\title{
Thalamic Shape Abnormalities in Individuals with Schizophrenia and Their Nonpsychotic Siblings
}

\author{
Michael P. Harms, ${ }^{1}$ Lei Wang, ${ }^{1}$ Daniel Mamah, ${ }^{1}$ Deanna M. Barch, ${ }^{1,4}$ Paul A. Thompson, ${ }^{3}$ and John G. Csernansky ${ }^{1,2}$ \\ Departments of ${ }^{1}$ Psychiatry and ${ }^{2}$ Anatomy and Neurobiology and ${ }^{3}$ Division of Biostatistics, Washington University School of Medicine, and ${ }^{4}$ Department of \\ Psychology, Washington University, St. Louis, Missouri 63110
}

\begin{abstract}
Deficits in the volume of the thalamus have been observed in both individuals with schizophrenia and their nonpsychotic relatives. However, no studies to date have examined the underlying pattern of thalamic shape change in relatives of individuals with schizophrenia. This study examined the volume and shape of the thalamus in schizophrenia subjects, their siblings, and healthy control individuals. T1-weighted magnetic resonance scans were collected in a group of young subjects with schizophrenia (mean age, 23 years) and their nonpsychotic siblings ( $n=25$ pairs), and control subjects and their siblings $(n=40$ pairs). Thalamic surfaces were generated using high-dimensional brain mapping. A canonical weighting function was derived from the contrast between schizophrenia and control subjects and then used to generate a canonical shape score for all subjects. Maps of the estimated surface displacement between groups were also created to visualize the thalamic shape differences between groups. The thalamic canonical scores of the siblings of the schizophrenia probands were intermediate between the probands and healthy control subjects. These siblings also displayed an intermediate degree of the inward surface deformation of the anterior and posterior thalamus that was present between schizophrenia probands and controls. There was no main effect of group status on thalamic volume and no significant correlations of the structural measures with measures of psychopathology or cognitive function. Our results indicate that thalamic shape abnormalities are present in relatively young individuals with schizophrenia and their siblings. Inward deformation of the anterior and posterior regions of the thalamus represents a potential neuroanatomical endophenotype of schizophrenia.
\end{abstract}

Key words: schizophrenia; thalamus; MRI; sibling; endophenotype; high-dimensional brain mapping

\section{Introduction}

The pathogenesis of complex neuropsychiatric disorders, such as schizophrenia, is influenced by the interaction of multiple genetic loci, as well as epigenetic, environmental, and random factors. To better understand this complexity, researchers are searching for "endophenotypes" (i.e., quantitative biological markers that are associated with an illness, exhibit heritability, and can be related to variation in a small number of genes). Many hope that this approach to decomposing complex psychiatric disorders will facilitate the linkage of genetics to the clinical syndrome, as well as promote the development of new animal models and therapies for treatment (Gottesman and Gould, 2003; Braff et al., 2007).

Neuroanatomical abnormalities have been observed in both schizophrenia subjects and their nonpsychotic relatives. Decreases in the volume of the hippocampus and cerebral gray matter are among the most commonly reported gross anatomical changes, although such findings are not replicated in all studies

Received June 6, 2007; revised 0ct. 24, 2007; accepted 0ct. 25, 2007.

This work was supported by National Institutes of Health Grants P50 MH071616 and R01 MH056584. We thank the staff of the Administration/Assessment and Biostatistical Cores of the Conte Center for the Neuroscience of Mental Disorders at Washington University School of Medicine for collection of the clinical and imaging data and data management, and Jae Kim for assistance with the landmarking procedure. The template image volume was provided by Surgical Navigation Technologies (Boulder, (O).

Correspondence should be addressed to Dr. Michael P. Harms, Department of Psychiatry, Box 8134, Washington University School of Medicine, 660 South Euclid Avenue, St. Louis, M0 63110. E-mail: mharms@conte.wustl.edu. D0I:10.1523/JNEUROSCI.2571-07.2007

Copyright $\odot 2007$ Society for Neuroscience $\quad 0270-6474 / 07 / 2713835-08 \$ 15.00 / 0$ (for review and meta-analysis, see Boos et al., 2007). The magnitude of such neuroanatomical abnormalities in nonpsychotic relatives tends to be intermediate between individuals with the disease and healthy controls even if not statistically different from both groups. Interestingly, such abnormalities have also been associated with obstetric complications (Cannon et al., 2002; van Erp et al., 2002; Schulze et al., 2003), which suggests that environmental insults may interact with genetic influences in their development.

In the current study, we assessed the volume and shape of the thalamus in subjects with schizophrenia and their nonpsychotic siblings. The thalamus was selected to be the focus of this study because of the central role it plays in the development of the cerebral cortex (Lopez-Bendito and Molnar, 2003). In fact, previous hypotheses of the pathogenesis of schizophrenia have suggested that neuroanatomical abnormalities of the cerebral cortex in schizophrenia could arise secondary to defects in thalamic neurogenesis (Selemon et al., 2005). Reductions in the total volume of the thalamic complex have been reported in schizophrenia subjects (for review, see Konick and Friedman, 2001; Sim et al., 2006). Moreover, postmortem and neuroimaging studies of individual thalamic nuclei suggest specific involvement of the anterior nucleus, mediodorsal nucleus, and pulvinar, all of which project to frontotemporal regions of the cortex (Pakkenberg, 1992; Young et al., 2000; Byne et al., 2002; Kemether et al., 2003; Danos et al., 2005). Previously, we reported abnormalities of tha- 
lamic shape in schizophrenia subjects relative to healthy controls, and found deformities in the anterior and posterior extremes of the structure in keeping with involvement of the anterior-most nuclei and pulvinar (Csernansky et al., 2004).

Given the apparently localized nature of thalamic abnormalities in schizophrenia subjects, quantitative analysis of the thalamic surface has the potential to identify a specific endophenotype of thalamic structural changes associated with schizophrenia. Decreases in thalamic volume of $\sim 5 \%$ have been reported in the relatives of schizophrenia subjects compared with healthy controls (Staal et al., 1998; Seidman et al., 1999; Lawrie et al., 2001). However, no studies to date have examined the underlying pattern of thalamic shape change in relatives of individuals with schizophrenia. Here, we report that the nonpsychotic siblings of individuals with schizophrenia exhibit a degree of thalamic surface change intermediate between schizophrenia and controls, and that these changes are most prominent in the same anterior and posterior regions where individuals with schizophrenia differ most from control subjects.

\section{Materials and Methods}

Subjects. The subjects for this study were recruited through the Conte Center for the Neuroscience of Mental Disorders (CCNMD) at Washington University in St. Louis and included (1) individuals with schizophrenia based on Diagnostic and Statistical Manual of Mental Disorders, Ed IV (DSM-IV) criteria (SCZ), (2) their nonpsychotic siblings (SCZ$\mathrm{SIB})$; (3) healthy control participants (CON), and (4) their siblings (CON-SIB). Siblings were full siblings, based on self report. The study was approved by the Washington University, St. Louis, Institutional Review Board, and all subjects gave their written informed consent after a complete description of the study.

Demographic information for the four groups of subjects is contained in Table 1. All subjects were diagnosed using DSM-IV criteria on the basis of a consensus between a research psychiatrist who conducted a semistructured interview and a trained research assistant who used the Structured Clinical Interview for DSM-IV Axis I Disorders (First et al., 2001). Participants from any of the four groups were excluded if they (1) met DSM-IV criteria for current substance abuse or dependence (i.e., during the month preceeding assessment), (2) had a clinically unstable or severe medical disorder, or a medical disorder that confounded the assessment of psychiatric diagnosis or rendered research participation dangerous, (3) had a head injury (past or present) with documented neurological sequelae or loss of consciousness, or (4) met DSM-IV criteria for mental retardation (mild or greater in severity).

SCZ participants were recruited from local inpatient and outpatient treatment facilities, and were stabilized on antipsychotic medication for at least 2 weeks before participating in the study (reported antipsychotic medication during the 4 weeks before clinical assessment was one of the following: atypical antipsychotics, $n=20$; typical, $n=1$; combination of atypical and typical, $n=2$; unknown because of participation in a blinded medical trial, $n=2$ ). CON participants were recruited using local advertisements in the same community, and were required to have no lifetime history of Axis I psychotic or major mood disorders (e.g., major depressive disorder and bipolar disorder) and no first-degree relatives with a psychotic disorder. Such requirements are common for the "healthy" comparison group of psychiatric studies. Potential SCZ-SIB subjects were excluded if they had a lifetime history of any DSM-IV Axis
I psychotic disorder, but not other DSM-IV Axis I disorders. Therefore, to control for possible effects arising from psychiatric disorders other than schizophrenia, the siblings of healthy comparison subjects were included as an additional comparison group. The CON-SIB subjects were enrolled in an identical manner to the SCZ-SIB subjects, and met the same general and specific inclusion and exclusion criteria.

Clinical and cognitive assessments. Psychopathology and cognitive function were assessed in a manner similar to that described by Delawalla et al. (2006). Briefly, psychopathology was assessed using the Scale for the Assessment of Negative Symptoms (SANS), the Scale for the Assessment of Positive Symptoms (Andreasen et al., 1995), the Structured Interview for Prodromal Syndromes (Miller et al., 1999), and the Chapman Psychosis Proneness Scales (Chapman et al., 1995). Cognitive function was assessed using a battery of neuropsychological tests. The raw scores from the individual psychopathological and neuropsychological tests were first $\mathrm{z}$-scored using the mean and SD computed across all subjects who have participated in sibling research studies at our CCNMD (216 subjects: 47 SCZ, 40 SCZ-SIB, 63 CON-SIB, 66 CON), and selected clusters of $z$-scores were then averaged to yield three domains of clinical symptoms (positive symptoms, negative symptoms, and thought disorganization) and four broad cognitive domains (working memory, episodic memory, executive function, and attention). These clinical symptoms and cognitive domain clusters were composed of the same items as in Delawalla et al. (2006) except that (1) the score for attention from the SANS was moved from the negative symptom cluster to the thought disorganization cluster, (2) the four-item d' score from the continuous performance task and the scaled score for spatial span from the Wechsler Memory Scale were added to the working memory domain, and (3) the score for perseverative errors (reversed in sign) from the Wisconsin Card Sort was added to the executive function domain. These modifications were made based on analyses of internal consistency, as assessed by Cronbach's $\alpha$, in a larger sample of subjects than was available for Delawalla et al. (2006). The resulting alphas for positive symptoms, negative symptoms, and thought disorganization were $0.88,0.91$, and 0.73 , respectively. For the working memory, episodic memory, executive function, and attention domains the alphas were $0.82,0.77,0.73$, and 0.73 , respectively.

Image collection and preparation. Magnetic resonance (MR) scans of the whole brain were collected using a Siemens (Erlangen, Germany) Magnetom Vision 1.5T imaging system using a standard head coil and a three-dimensional FLASH sequence (repetition time, $20 \mathrm{~ms}$; echo time, 
Table 2. Adjusted thalamic and cerebral volumes (cubic centimeters)

\begin{tabular}{lllll}
\hline & SCZ & SCZ-SIB & CON-SIB & CON \\
\hline $\begin{array}{l}\text { Thalamus } \\
\text { Adjusted for gender }\end{array}$ & & & & \\
$\quad$ L & $7.21(0.14)$ & $7.60(0.11)$ & $7.66(0.10)$ & $7.60(0.09)$ \\
$\quad$ R & $7.41(0.16)$ & $7.65(0.13)$ & $7.72(0.11)$ & $7.70(0.09)$ \\
$\quad$ Adjusted for gender and cerebral volume & & & & $7.49(0.07)$ \\
$\quad$ L & $7.49(0.11)$ & $7.74(0.10)$ & $7.49(0.08)$ & $7.58(0.07)$ \\
$\quad$ R & $7.69(0.13)$ & $7.78(0.12)$ & $7.56(0.08)$ & $1011(14.4)$ \\
Cerebral volume & & & & \\
$\quad$ Adjusted for gender & $916(19.9)$ & $952(15.6)$ & $1024(14.3)$ & $1010)$ \\
\hline
\end{tabular}

Values are the predicted marginal means, or "least square means," adjusted for the indicated effects. There was a main effect of group on cerebral volume $\left(F_{(3,46)}=6.3 ; p=0.001\right)$, but not thalamic volume. Values in parentheses are SEs. L, Left; $R$, right.

$5.4 \mathrm{~ms}$; flip angle, $30^{\circ}$; number of acquisitions, 1 ; voxel size, $1 \times 1 \times 1$ $\mathrm{mm}^{3}$; scanning time, $13.5 \mathrm{~min}$ ). Signal intensity differences across subjects were normalized by linear rescaling, using the intensity of the corpus callosum and the third ventricle as reference structures (Wang et al., 2007).

Generation of the thalamic surfaces. We followed previously established and validated procedures to generate thalamic surfaces in each individual subject (Csernansky et al., 2004; Mamah et al., 2007; Wang et al., 2007). First, a template MR scan (magnetization-prepared rapid acquisition gradient echo) was collected in a healthy control subject that was not otherwise included in the analysis. The right thalamus in the template scan was outlined manually and a template thalamic surface was generated by superimposing a triangulated mesh of points onto its external boundary. Next, in the template scan and in the scans of all subjects in the study, landmarks were placed at the external boundaries of the brain (anterior, posterior, superior, inferior, and lateral boundaries), the intersection of the anterior and posterior commissures with the midsagittal plane, and at preselected points within and around the complex of the left and right thalamus and basal ganglia (Wang et al., 2007). The thalamus and basal ganglia were landmarked together to promote efficient generation of surfaces for these adjacent structures; results for the basal ganglia will be reported elsewhere. Finally, the template thalamic surface was mapped onto the left and right sides of each subject in a two-step process that first used the landmarks to provide "anchor" points for initial scan registration. This initial registration was then followed by largedeformation high-dimensional brain mapping (Haller et al., 1997; Miller et al., 1997). By mapping the left and right thalamus in all subjects using the same template thalamic surface, a correspondence of surface points was maintained across all subjects and both hemispheres.

Measurements of total cerebral volume (excluding the ventricles, brainstem, and cerebellum) were obtained from FreeSurfer, a semiautomated software package for segmentation and cortical surface reconstruction (Dale et al., 1999), so that total cerebral volume could be included as a covariate in statistical analyses.

Data analysis. Principal components analysis (PCA) was used to reduce the high dimensionality of the datasets representing the left and right thalamic surfaces (4790 surface vertices per subject per hemisphere). Before PCA, the left and right thalamic surfaces of all subjects were brought into a common coordinate space via rigid-body registration (translation and rotation, including reflection for the left surfaces). The columns of the matrix for PCA were obtained by representing the surface coordinates of each thalamus as a column vector. The thalamic surfaces from all subjects with useable MR data at our CCNMD were included in the derivation of the principal component directions [i.e., 330 surfaces representing 165 subjects (37 SCZ, 31 SCZ-SIB, 49 CON$\mathrm{SIB}, 48 \mathrm{CON}$ )]. However, in the subsequent analyses we used only the PC scores from individuals for whom useable MR data were also available from their sibling (130 total subjects) (Table 1) because we planned to examine the correlations between neuroanatomical variables within sibling pairs. Each thalamic surface was projected into the space defined by the first 15 principal components (PCs), yielding 15 PC scores for each thalamic surface (left and right; 30 scores total per subject), which accounted for $88 \%$ of total shape variance (across subjects and hemispheres). Multivariate ANOVA (MANOVA) was performed using the
PC scores from all four subject groups, with hemisphere treated as a repeated factor, and gender included as a covariate, to examine whether there were overall differences in thalamic shape across groups or hemisphere.

Next, to test our hypothesis of an ordered variation in thalamic shape across the four subject groups, we computed a single set of 15 PC scores for each subject by averaging corresponding PC scores from the left and right thalamus, and applied canonical analysis to the averaged PC scores to generate canonical scores. The canonical analysis was designed to score the SCZ-SIB and CON-SIB subjects along the dimension that "maximized" the difference the SCZ and CON subjects. More specifically, using a general linear model with the PC scores as dependent variables, and group and gender as predictor variables, the canonical analysis computed the first eigenvector of $\left(\mathbf{E}^{-1}\right) \times \mathbf{H}$, where $\mathbf{H}$ was the sum of squares and cross-products (SSCP) matrix associated with the contrast between the SCZ and CON subjects, and $\mathbf{E}$ was the SSCP matrix of the model residuals (derived from the full model using all subjects). The eigenvector contained the weighting coefficients for computing the canonical scores. A canonical score was obtained for each subject by applying these weighting coefficients to the original dependent variables (i.e., the PC scores).

Group differences in cerebral and thalamic volume, thalamic canonical scores, and the clinical and cognitive domain scores were assessed using mixed-model methods that explicitly estimated the covariance (correlation) in the residuals attributable to the sibling relationships. For thalamic volume, the mixed-model additionally included the correlation across hemispheres as part of the covariance structure. Gender was included as a covariate (i.e., additional fixed effect predictor) for all analyses of volume and canonical scores. Additional analyses of volume and canonical scores included either (1) total cerebral volume or (2) age, duration of illness (coded as zero for the subjects from the three nonpsychotic groups), and lifetime history of substance dependence, mood disorders, or anxiety disorders (each coded as a separate effect) as additional covariates. Because the canonical scores were derived from a contrast between the SCZ and CON groups, planned comparisons for the canonical scores were restricted to SCZ versus SCZ-SIB, SCZ-SIB versus CONSIB, SCZ-SIB versus CON, and CON-SIB versus CON. In all mixed model analyses, the covariance structure was "unstructured," the two different sibling pairs were allowed to be heterogeneous in their covariance estimates, and the degrees of freedom calculations used the method of Kenward and Roger (1997). The significance of the predictor variables was assessed using type III sums-of-squares. All statistical analyses were performed in SAS 9.1 (SAS Institute, Cary, NC).

\section{Results}

\section{Thalamic and cerebral volume}

Thalamic volume was compared across the four groups of subjects using a mixed linear model that specified group, hemisphere, the interaction of group with hemisphere, and gender as fixed effects. There was a significant effect of hemisphere on thalamic volume $\left(F_{(1,50)}=4.4 ; p=0.04\right)$, but no effect of group status $\left(F_{(3,52)}=1.8 ; p=0.16\right)$ or group by hemisphere interaction $\left(F_{(3,59)}=0.6 ; p=0.6\right)$. Similar results were obtained when total cerebral volume was included as a covariate. Cerebral volume itself varied across groups $\left(F_{(3,46)}=6.3 ; p=0.001\right)$, as assessed by a mixed model with group and gender as fixed effects. Post hoc tests indicated that cerebral volume in the SCZ-SIB was larger than the SCZ ( $p=0.05)$, but smaller than the CON and CON-SIB subjects ( $p=0.008$ and 0.001 , respectively). Cerebral volume in the CON and CON-SIB subjects did not differ ( $p=$ $0.4)$. Estimated thalamic and cerebral volumes are shown in Table 2 . 


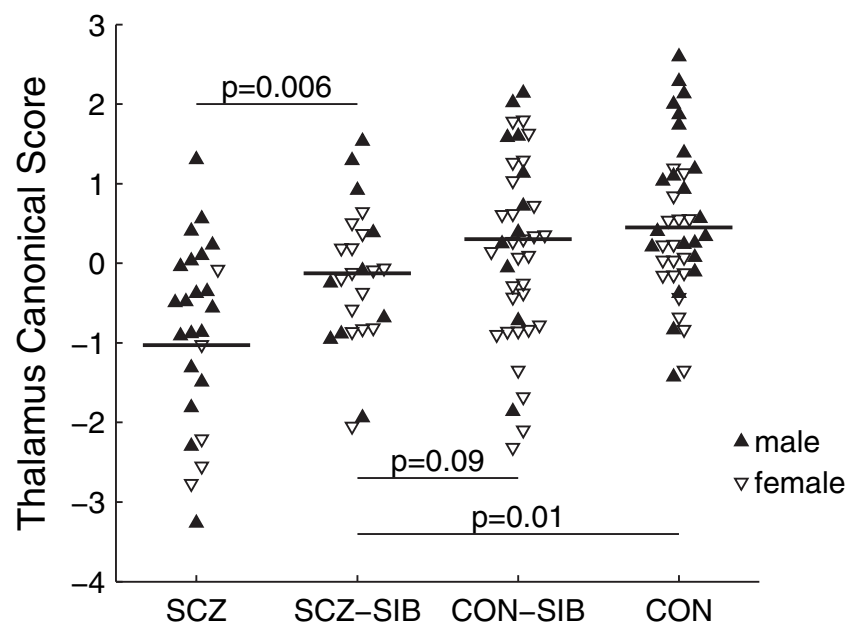

Figure 1. Scatterplot of thalamic canonical scores for the four subject groups. Canonical analysis was used to establish the linear combination of PC scores that maximized the difference between the SCZ and CON subjects. The resulting weighting coefficients were then used to score all subjects. Differences between groups were assessed using a mixed model with group and gender as fixed effects. Horizontal line indicates the predicted marginal mean ("least square mean") controlling for gender [least square means (SEs) of $-1.03(0.22),-0.13(0.18), 0.30$ (0.18), and $0.45(0.14)$ for SCZ, SCZ-SIB, CON-SIB, and CON, respectively].

\section{Thalamic shape}

MANOVA applied to the PC scores summarizing thalamic surface variation, with hemisphere as a repeated factor and gender as a covariate, indicated an effect of group status $\left(F_{(45,331)}=1.4 ; p=\right.$ 0.05 , Wilks' Lambda) and hemisphere $\left(F_{(15,11)}=7.5 ; p<\right.$ $0.0001)$. The interaction between group and hemisphere was not significant $\left(F_{(45,331)}=0.9 ; p=0.6\right)$. Importantly, the multivariate contrast between the SCZ and CON groups was significant $\left(F_{(15,111)}=2.0 ; p=0.03\right)$. This result provided a basis for a canonical analysis to compare univariate canonical scores of the SCZ-SIB and CON-SIB to the other groups. This strategy maximized the power to detect differences between groups (as defined by the SCZ vs CON difference).

We tested for an ordered variation in the thalamic surfaces of the SCZ-SIB and CON-SIB subjects relative to the SCZ and CON subjects by deriving a canonical score for each subject using the linear combination of PC scores that maximized the difference between the SCZ and CON subjects (see Materials and Methods). The mean canonical scores by group exhibited a progression from SCZ to SCZ-SIB to CON-SIB to CON (Fig. 1). Mixed model analysis of the canonical scores with group and gender as fixed effects indicated that the SCZ-SIB scores were statistically greater than the SCZ subjects $\left(F_{(1,26)}=9.1 ; p=0.006\right)$, but less than the CON subjects $\left(F_{(1,51)}=6.4 ; p=0.01\right)$. The difference between SCZ-SIB and CON-SIB subjects also trended toward significance $\left(F_{(1,59)}=2.9 ; p=0.09\right)$. Canonical scores for the CON-SIB and CON subjects did not differ $(p=0.5)$.

When variables coding for age, duration of illness, and lifetime history of substance dependence, mood disorders, and anxiety disorders were included as additional fixed effects in the mixed models for thalamic canonical score, thalamic volume and cerebral volume, none of these covariates predicted any of these three structural measures $(p>0.14)$. Furthermore, in an analysis restricted to the SCZ subjects, there was no correlation of either age or illness duration with thalamic canonical score, total thalamic volume, or cerebral volume (Pearson's $r<0.14 ; p>0.5$ ). The correlation of age and illness duration in the SCZ subjects was $r=0.66$.
The pattern of thalamic shape variation between groups was visualized by estimating the displacement of the thalamic surfaces between different subject groups, while controlling for gender (Fig. 2A). Maps highlighting the regions of largest displacement (relative to the variance) were also computed for each comparison (Fig. $2 B$ ) by thresholding the $p$ value of the pairwise group difference at each surface node at $p=0.05$ (uncorrected for multiple comparisons). For the SCZ versus CON comparison, there was an inward deformation at the anterior and posterior extremes of the thalamus, which was larger in the left hemisphere than the right. The SCZ-SIB versus CON and SCZ versus SCZSIB comparisons together illustrate that SCZ-SIB subjects exhibit a degree of surface displacement intermediate between $C O N$ and SCZ subjects. Namely, the SCZ-SIB subjects exhibited inward displacement at the anterior and posterior of the thalamus relative to CON subjects (as well as CON-SIB subjects, data not shown), and the SCZ subjects exhibited additional inward displacement relative to the SCZ-SIB subjects, particularly in the anterior of the left thalamus and in a penumbra around the posterior-most region of the left thalamus. In contrast, surface differences in the CON-SIB versus CON comparison were muted in these same anterior and posterior regions.

To assess the relationship between the canonical scores generated for each subject and the observed pattern of surface deformation, we computed the correlation of the canonical scores of the SCZ and CON subjects with the surface displacements of those same subjects, and displayed the magnitude of these correlations on the thalamic surface (Fig. 3). Consistent with the idea that the canonical scores capture a dimension relevant to the surface displacements between SCZ and CON subjects, the largest correlations occurred at the anterior and posterior portions of the thalamus (i.e., in approximately the same regions that exhibited the largest deformations between the SCZ and CON subjects). The direction of this correlation was positive, indicating a correlation in those regions between outward surface displacements and higher (i.e., more CON-like) canonical scores. In a similar manner, we also computed analogous maps (data not shown) for each group separately and found strong positive correlation $(r>0.5)$ between canonical scores and surface displacements at the anterior of the thalamus for each of the four groups, and at the posterior of the thalamus for the SCZ, CON-SIB, and CON groups. (The SCZ-SIB group had a positive correlation between these measures along a portion of the right posteriosuperiolateral surface, a region that likely still overlies the pulvinar and which exhibited positive correlation in the correlation maps of the other three groups as well, but not at the posteriormost portion of the left and right thalamus as was the case in the other three groups.) Overall, these correlation maps indicate that the anterior and posterior extremes of the thalamus were the regions with the strongest coupling between surface displacements and variations in canonical scores.

\section{Relationships of structural, clinical, and neuropsychological measures}

The clinical and neuropsychological scores for the four subject groups are plotted in Figure 4. On all three of the clinical domains (positive, negative, and disorganization symptoms) and three of the four cognitive domains (episodic memory, working memory, and executive function), the siblings of schizophrenia subjects exhibited an intermediate degree of clinical symptoms and cognitive deficits relative to the schizophrenia probands and healthy controls.

The correlations of the structural measures with the clinical 


\section{Estimated surface displacement between groups}

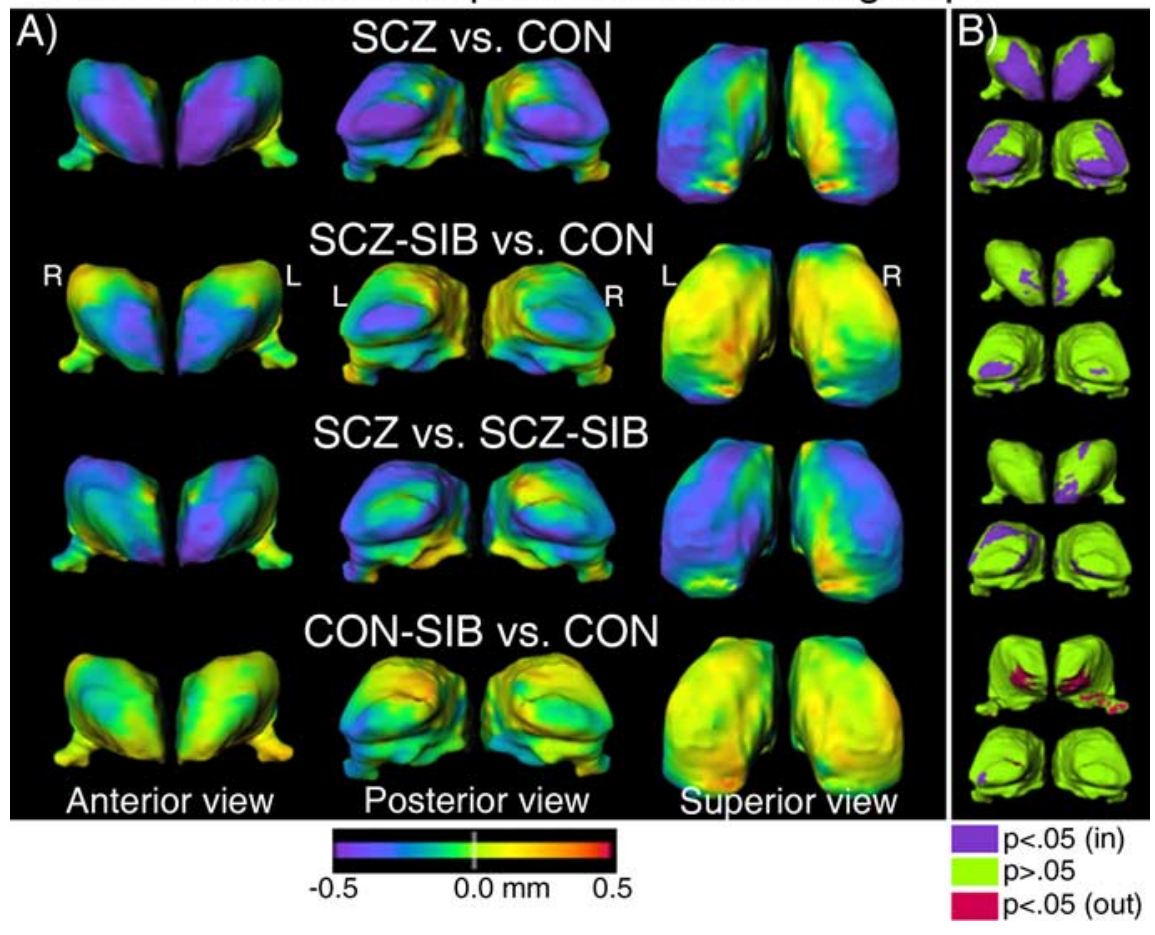

Figure 2. A, Maps of the estimated displacement between different subject groups, controlling for gender. These were obtained by first computing for every thalamus the surface-normal component of the displacement of each surface node of that thalamus relative to the average thalamic surface of all 130 subjects. Then, we computed the least square mean of these displacements for each group (and surface node) controlling for gender. Last, for each pairwise group comparison shown, the difference of these least square means between the two selected groups was displayed as a color map, with cool (warm) colors indicating inward (outward) displacement relative to the second group listed in each comparison (i.e., SCZ-SIB group for the third row, $\mathrm{CON}$ group for all other rows). The reference surface on which the color maps are overlaid is the mean surface of the second group listed in each comparison. For the SCZ versus CON comparison, the inward displacements at the anterior and posterior regions of the thalamus exceeded the limit of the color scale, particularly on the left hemisphere (largest inward displacement was $0.86 \mathrm{~mm}$ in the anterior-most region of the left thalamus), such that the larger displacements in the left thalamus (vs the right) are not well visualized. For the superior view, the anterior-to-posterior direction is upward. $\boldsymbol{B}$, Thresholded maps highlighting the regions of largest displacement (relative to the variance), assessed by computing at each node the $p$ value associated with the difference in least square means used in constructing the comparison in $\boldsymbol{A}$ (i.e., gender included as a covariate). Surface nodes having an inward displacement for which $p<0.05$ (not adjusted for multiple comparisons) are shown in purple and nodes with $p>0.05$ in green. Only a few small patches of the surfaces exhibited outward displacements with $p<0.05$ (shown in red). The anterior of the thalamus in the CON-SIB versus CON comparison in $B$ is tilted upward to reveal the location of the outward displacement of largest surface area of all four comparisons. The anterior and posterior views shown capture all of the main regions for which $p<0.05$. L, Left; $R$, right.

and cognitive domain scores were assessed within a combined group of SCZ and SCZ-SIB subjects. The largest correlation (assessed using nonparametric Spearman's $r$ ) of total cerebral volume, left or right thalamic volume, or thalamic canonical score with any of the four cognitive domain scores or three clinical symptom scores was a weak correlation between canonical score and working memory (Spearman's $r=0.27, p=0.06$; all other $r$ values $<0.21, p>0.14$ ). Analysis of the SCZ and SCZ-SIB groups separately indicated that this weak correlation between thalamic canonical score and working memory was driven by the SCZ-SIB subjects $(r=0.28)$ because it was absent in the SCZ subjects $(r=$ $0.06)$. As an additional exploratory analysis in the combined group of SCZ and SCZ-SIB subjects we computed the canonical correlation of the four structural measures against either the four cognitive or three clinical measures to determine the highest possible correlation between any linear combination of one set of variables against the other. In both cases, the canonical correlation accounted for $<25 \%$ of the variance along the dimension of the first canonical variables and the canonical correlations were not significant, indicating that there was no evidence of a relationship between some linear combination of the four structural measures with a linear combination of either the cognitive or clinical domains (structural vs cognitive variables, first canonical correlation $\rho=0.48, p=0.2$; structural vs clinical variables, $\rho=0.41$, $p=0.5$ ).

Correlating the structural measures to each other (in the combined group of SCZ and SCZ-SIB subjects) we found, as anticipated, that cerebral volume and the volume of the left and right thalamus were all correlated with each other (Spearman's $r$ $>0.63$; $p<0.0001)$. The thalamic canonical scores were correlated with these other three structural measures to a lesser degree $(r=0.35-0.47 ; p<0.01)$. Last, we examined the correlation between siblings of the residuals of the four structural measures after removing the effect of gender. Cerebral volume was positively correlated between siblings in both pairs of siblings (Table 3 ). Left and right thalamic volumes were positively correlated between siblings in only the SCZ/SCZ-SIB sibling pair, and the difference in correlation coefficients between the two groups of sibling pairs reached significance for left thalamic volume ( $p=$ 0.03, assessed using Fisher's $z$ transformation to compare correlation coefficients). Thalamic canonical scores were not correlated in either pair of siblings.

\section{Discussion}

We found evidence for abnormalities in the shape of the thalamus, as well as altered cognitive performance and clinical symptomatology, in the nonpsychotic siblings of individuals with schizophrenia. This result is in keeping with a large literature suggesting that family members of individuals with schizophrenia have an increased risk of developing the disorder (Gottesman, 1991), electrophysiological deficits (Clementz et al., 1998; Cadenhead et al., 2000), abnormal brain activation (Callicott et al., 2003; Fusar-Poli et al., 2007), and a variety of structural brain abnormalities (Boos et al., 2007). Such measures represent potential "endophenotypes" that may help clarify the relationship between genetic variation and the clinical symptoms of schizophrenia (Gottesman and Gould, 2003; Braff et al., 2007).

Although the primary focus of our study was to determine whether differences in thalamic shape exist between schizophrenia subjects, their siblings, and healthy controls, we naturally examined overall thalamic volume as well. There was no effect of group status on thalamic volume, regardless of whether cerebral volume was included as a covariate. Previously, we found a thalamic volume reduction of $7 \%$ in a group of more chronic schizophrenia subjects (mean age, 38 years) relative to age-matched healthy controls, which became nonsignificant after including cerebral volume as a covariate (Csernansky et al., 2004). Although a meta-analysis by Konick and Friedman (2001) provided 


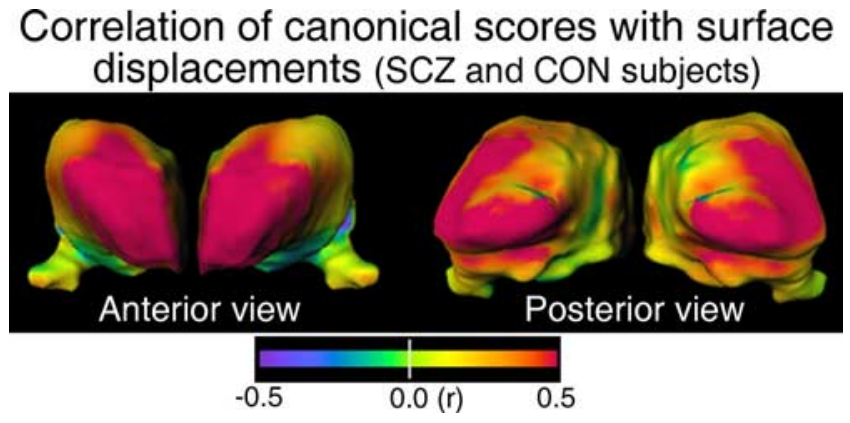

Figure 3. Maps of the Pearson correlation of canonical scores in the SCZ and CON subjects with surface displacements in those same subjects. A surface-normal displacement map was computed for each individual subject (relative to the average surface of all subjects) with outward displacements positive, and inward displacements negative. The correlation of these displacements with the canonical scores was computed at each surface node.

evidence for a small-to-moderate reduction of thalamic volume in schizophrenia (Cohen's $d \sim 0.3$ ), even after normalization by brain or cranial volume, the individual studies included in this meta-analysis frequently failed to find significant differences. Not surprisingly then, the literature regarding whether the nonpsychotic relatives of individuals with schizophrenia exhibit volume deficits in the thalamus is also ambiguous. Several studies have reported statistically significant decreases in thalamic volume in relatives compared with controls (Staal et al., 1998; Seidman et al., 1999; Lawrie et al., 2001). However, Seidman et al. (1999) did not have MR scans for the schizophrenia probands, and Lawrie et al. (2001) reported lower mean thalamic volume in the relatives than the schizophrenia probands (who did not differ from controls). More recently, using voxel-based morphometry, McDonald et al. (2004) found that genetic risk for schizophrenia was associated with decreased gray matter volume density in the thalamus of schizophrenia probands and their nonpsychotic relatives. However, Ettinger et al. (2007) found no significant differences in thalamic volume between controls and monozygotic twins discordant for schizophrenia. We likewise found no evidence for detectable changes of thalamic volume in siblings of schizophrenia subjects relative to controls. Rather, our results indicate that abnormalities of the thalamus may be confined to localized subregions, and that increased power for detecting thalamic volume abnormalities in schizophrenia subjects and their relatives may be achieved by focusing on such subregions of the thalamus.

To check for possible localized variations in thalamic shape that are not captured by volume measures, we used two complementary approaches. In one approach, we derived a canonical score for each subject and found that the mean canonical score for SCZ-SIB subjects was intermediate between SCZ and CON subjects. In the second approach, we created maps of the surface deformation between groups. These maps showed that both the SCZ and SCZ-SIB exhibited inward deformation at the anterior and posterior extremes of the thalamus relative to CON subjects, with SCZ-SIB having a degree of surface displacement intermediate between CON and SCZ subjects. We linked these two approaches by showing that the largest correlation between canonical scores and surface displacement occurred at the anterior and posterior extremes of the thalamus. Notably, the pattern of thalamic shape change in the schizophrenia subjects and their siblings was similar to what we previously observed in a nonoverlapping group of schizophrenia subjects compared with healthy controls (Csernansky et al., 2004). Overall, our results indicate

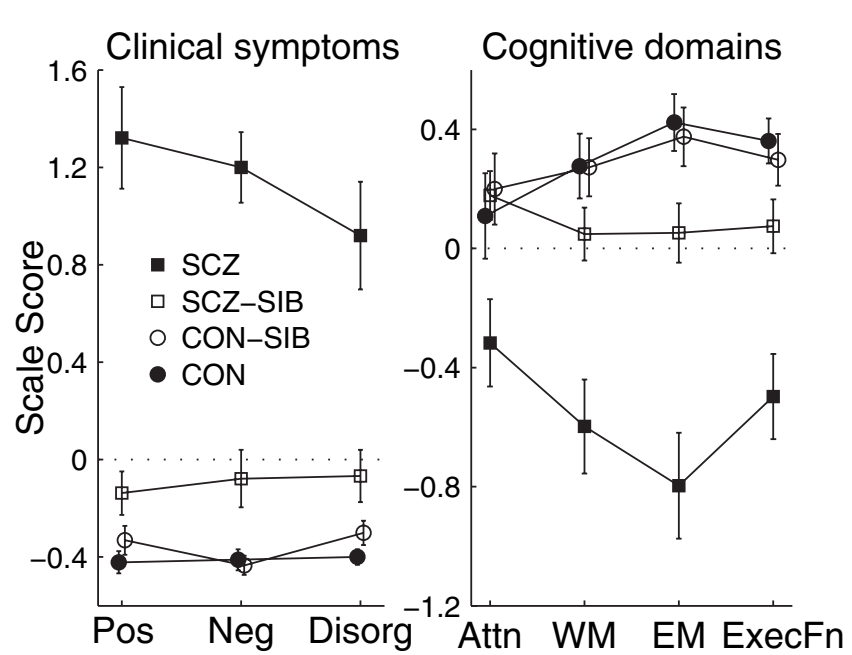

Figure 4. Plots of mean Z-score ( \pm SE) for the clinical symptoms (positive, negative, and disorganization symptoms) and cognitive domains (attention, working memory, episodic memory, and executive function) for the complete pair siblings whose demographics are provided in Table 1. There was a significant main effect of group for all seven scores $(F>3.3 ; p<$ 0.03 , mixed model). The difference between SCZ and SCZ-SIB subjects was significant for all seven scores $(F>9.3 ; p<0.01)$. The difference between SCZ-SIB and CON (or CON-SIB) subjects was significant (or nearly so) for episodic memory, executive function, and all three clinical symptoms (SCZ-SIB vs CON, $F>5.9, p<0.02 ;$ SCZ-SIB vs CON-SIB, $F>3.3, p<0.08$ ). The CON and CON-SIB subjects did not differ on any of the seven scores. Similar results were obtained when gender was included as a covariate (the gender effect was not significant for any of the scores, $p>0.15$ ).

Table 3. Spearman correlation coefficients between siblings (SCZ vs SCZ-SIB; CON vs CON-SIB) for the structural measures, controlling for gender

\begin{tabular}{lccccc}
\hline & \multicolumn{2}{c}{ SCZ vs SCZ-SIB } & & \multicolumn{2}{c}{ CON vs CON-SIB } \\
\cline { 2 - 3 } & $r$ & & & $r$ & $p$ \\
\hline Cerebral volume & 0.51 & 0.01 & & 0.30 & 0.06 \\
Left thalamic volume* $^{*}$ & 0.59 & 0.002 & & 0.09 & 0.6 \\
Right thalamic volume & 0.51 & 0.009 & & 0.18 & 0.3 \\
Thalamic canonical score & -0.07 & 0.7 & & -0.06 & 0.7 \\
\hline
\end{tabular}

For each structural measure, Spearman correlations between the siblings of each sibling pair were computed on the residuals after regressing out the effect of gender (estimated using all subjects so that there was a single estimate of the gender effect). $n=25$ for SCZ versus SCZ-SIB; $n=40$ for CON versus CON-SIB. *Significant difference ( $p=$ 0.03 ) in the correlation coefficients between the two sets of siblings (using Fisher's $z$ transformation).

that siblings of schizophrenia subjects have an attenuated form of the thalamic shape abnormalities present in individuals with schizophrenia.

The pattern of thalamic shape deformations in the schizophrenia probands and their siblings suggests involvement of the anterior nuclei and pulvinar of the thalamus, because these are the nuclei directly underlying the anterior and posterior thalamus. Postmortem and neuroimaging studies of individual thalamic nuclei or regions have indicated reduced volume of the pulvinar in schizophrenia (Byne et al., 2002; Kemether et al., 2003) and varying degrees of reduced volume of the anterior nucleus (Young et al., 2000; Byne et al., 2006) or the anterior region of the thalamus (Hazlett et al., 1999; Gilbert et al., 2001). Nonetheless, our thalamic surface deformations could be a consequence of volume loss in a variety of nuclei along the overall anterior-posterior axis of the thalamus. Identifying individual thalamic nuclei in vivo will require the use of image processing approaches designed to enhance nuclear boundaries (Kemether et al., 2003) or enhanced imaging protocols such as diffusion tensor imaging, which may provide a connectivity-based seg- 
mentation of thalamic gray matter (Behrens et al., 2003; Wiegell et al., 2003).

The presence of thalamic shape abnormalities in the siblings of schizophrenia probands suggests that genetic influences contribute to their development because twin and adoption studies have indicated that shared environmental factors have a negligible contribution to the overall etiology of schizophrenia (Kendler et al., 1994; Cannon et al., 1998; Cardno et al., 1999). However, shared environmental factors could nonetheless have a nonnegligible contribution to abnormalities observed within particular cerebral structures. Using variance components analysis, hippocampal volume was in fact found to be substantially influenced by unique and shared environmental factors in schizophrenia subjects and their nonpsychotic twins, whereas additive genetic effects dominated in healthy twin pairs (van Erp et al., 2004). We are not aware of any twin studies that have used similar analytic techniques in the thalamus. Thus, additional studies are necessary to quantify the relative contributions of genetic and environmental factors to thalamic volume and shape changes in schizophrenia.

The potential influence of antipsychotic medication on cerebral morphometry is a confounding factor in many neuroimaging studies of schizophrenia. In the present study, almost all of the schizophrenia subjects were taking second generation antipsychotic medication, which some studies have associated with thalamic enlargement (Gur et al., 1998; Dazzan et al., 2005). However, in other studies, thalamic enlargement has been more associated with first generation antipsychotic medications (Sullivan et al., 2003; Khorram et al., 2006). Regardless, our finding of thalamic shape changes in the siblings of individuals with schizophrenia strongly suggests that abnormalities of thalamic structure in schizophrenia cannot be solely explained as a consequence of antipsychotic drug treatment.

Our inclusion of siblings of control subjects as an additional comparison group is somewhat unique. CON subjects were recruited to be free of major mood disorders although their siblings were allowed to have a history of such conditions. Thus, the CON-SIB represent a less selected group of comparison subjects, who were more closely matched to the SCZ-SIB in terms of their history of nonpsychotic Axis I disorders. The canonical scores of CON-SIB and CON did not differ statistically, although the distribution of the CON-SIB scores was shifted downward slightly toward the SCZ-SIB. Notably, the difference between SCZ-SIB and CON-SIB achieved significance under a one tailed $t$ test, which would be justified given our hypothesis that the canonical scores of the SCZ-SIB would be intermediate between the SCZ and CON-SIB (or CON) groups.

There were no significant correlations of thalamic volume or canonical score with any of the three clinical symptom or four cognitive function domains in a combined group of SCZ and SCZ-SIB subjects. Previously we also found a general lack of correlation of volume or shape measures with clinical symptoms or cognitive function in the thalamus, hippocampus, and basal ganglia (Csernansky et al., 2002, 2004; Andrews et al., 2006; Mamah et al., 2007). An absence of correlation between thalamic volume and clinical measures has also been reported in other studies (Gilbert et al., 2001; Ettinger et al., 2007). Although it is possible that the differences in thalamic shape do not have readily observable functional consequences, it is also possible that the functional significance of these differences is not captured by conventional measures of clinical symptomatology and cognitive performance. Nonetheless, there was an association between tha- lamic shape and the most prominent "functional" variable; namely, the presence or absence of the disease state.

In conclusion, we found that the siblings of individuals with schizophrenia have an attenuated form of the deformations in thalamic shape present in schizophrenia. There was inward deformation of the anterior and posterior thalamus in both groups of subjects (versus controls), suggesting particular involvement of the anterior nuclei and pulvinar. These localized thalamic abnormalities represent a potential endophenotype of schizophrenia that may be useful for bridging clinical studies of schizophrenia to both genetics and more detailed postmortem studies of cellular pathology. Better characterization of the thalamic abnormalities in schizophrenia also provides the necessary framework for additional study and interpretation of structural and functional disturbances of the thalamocortical network related to the disease.

\section{References}

Andreasen NC, Arndt S, Alliger R, Miller D, Flaum M (1995) Symptoms of schizophrenia. Methods, meanings, and mechanisms. Arch Gen Psychiatry 52:341-351.

Andrews J, Wang L, Csernansky JG, Gado MH, Barch DM (2006) Abnormalities of thalamic activation and cognition in schizophrenia. Am J Psychiatry 163:463-469.

Behrens TE, Johansen-Berg H, Woolrich MW, Smith SM, Wheeler-Kingshott CA, Boulby PA, Barker GJ, Sillery EL, Sheehan K, Ciccarelli O, Thompson AJ, Brady JM, Matthews PM (2003) Non-invasive mapping of connections between human thalamus and cortex using diffusion imaging. Nat Neurosci 6:750-757.

Boos HB, Aleman A, Cahn W, Pol HH, Kahn RS (2007) Brain volumes in relatives of patients with schizophrenia: a meta-analysis. Arch Gen Psychiatry 64:297-304

Braff DL, Freedman R, Schork NJ, Gottesman II (2007) Deconstructing schizophrenia: an overview of the use of endophenotypes in order to understand a complex disorder. Schizophr Bull 33:21-32.

Byne W, Buchsbaum MS, Mattiace LA, Hazlett EA, Kemether E, Elhakem SL, Purohit DP, Haroutunian V, Jones L (2002) Postmortem assessment of thalamic nuclear volumes in subjects with schizophrenia. Am J Psychiatry 159:59-65.

Byne W, Kidkardnee S, Tatusov A, Yiannoulos G, Buchsbaum MS, Haroutunian V (2006) Schizophrenia-associated reduction of neuronal and oligodendrocyte numbers in the anterior principal thalamic nucleus. Schizophr Res 85:245-253.

Cadenhead KS, Swerdlow NR, Shafer KM, Diaz M, Braff DL (2000) Modulation of the startle response and startle laterality in relatives of schizophrenic patients and in subjects with schizotypal personality disorder: evidence of inhibitory deficits. Am J Psychiatry 157:1660-1668.

Callicott JH, Egan MF, Mattay VS, Bertolino A, Bone AD, Verchinksi B, Weinberger DR (2003) Abnormal fMRI response of the dorsolateral prefrontal cortex in cognitively intact siblings of patients with schizophrenia. Am J Psychiatry 160:709-719.

Cannon TD, Kaprio J, Lonnqvist J, Huttunen M, Koskenvuo M (1998) The genetic epidemiology of schizophrenia in a Finnish twin cohort. A population-based modeling study. Arch Gen Psychiatry 55:67-74.

Cannon TD, van Erp TG, Rosso IM, Huttunen M, Lonnqvist J, Pirkola T, Salonen O, Valanne L, Poutanen VP, Standertskjold-Nordenstam CG (2002) Fetal hypoxia and structural brain abnormalities in schizophrenic patients, their siblings, and controls. Arch Gen Psychiatry 59:35-41.

Cardno AG, Marshall EJ, Coid B, Macdonald AM, Ribchester TR, Davies NJ, Venturi P, Jones LA, Lewis SW, Sham PC, Gottesman II, Farmer AE, McGuffin P, Reveley AM, Murray RM (1999) Heritability estimates for psychotic disorders: the Maudsley twin psychosis series. Arch Gen Psychiatry 56:162-168.

Chapman JP, Chapman LJ, Kwapil TR (1995) Scales for the measurement of schizotypy. In: Schizotypal personality (Raine T, Lencz T, Mednick S, eds), pp 79-106. New York: Cambridge UP.

Clementz BA, Geyer MA, Braff DL (1998) Poor P50 suppression among schizophrenia patients and their first-degree biological relatives. Am J Psychiatry 155:1691-1694.

Csernansky JG, Wang L, Jones D, Rastogi-Cruz D, Posener JA, Heydebrand 
G, Miller JP, Miller MI (2002) Hippocampal deformities in schizophrenia characterized by high dimensional brain mapping. Am J Psychiatry 159:2000-2006.

Csernansky JG, Schindler MK, Splinter NR, Wang L, Gado M, Selemon LD, Rastogi-Cruz D, Posener JA, Thompson PA, Miller MI (2004) Abnormalities of thalamic volume and shape in schizophrenia. Am J Psychiatry 161:896-902.

Dale AM, Fischl B, Sereno MI (1999) Cortical surface-based analysis. I. Segmentation and surface reconstruction. Neuroimage 9:179-194.

Danos P, Schmidt A, Baumann B, Bernstein HG, Northoff G, Stauch R, Krell D, Bogerts B (2005) Volume and neuron number of the mediodorsal thalamic nucleus in schizophrenia: a replication study. Psychiatry Res 140:281-289.

Dazzan P, Morgan KD, Orr K, Hutchinson G, Chitnis X, Suckling J, Fearon P, McGuire PK, Mallett RM, Jones PB, Leff J, Murray RM (2005) Different effects of typical and atypical antipsychotics on grey matter in first episode psychosis: the AESOP study. Neuropsychopharmacology 30:765-774.

Delawalla Z, Barch DM, Fisher Eastep JL, Thomason ES, Hanewinkel MJ, Thompson PA, Csernansky JG (2006) Factors mediating cognitive deficits and psychopathology among siblings of individuals with schizophrenia. Schizophr Bull 32:525-537.

Ettinger U, Picchioni M, Landau S, Matsumoto K, van Haren NE, Marshall N, Hall MH, Schulze K, Toulopoulou T, Davies N, Ribchester T, McGuire PK, Murray RM (2007) Magnetic resonance imaging of the thalamus and adhesio interthalamica in twins with schizophrenia. Arch Gen Psychiatry 64:401-409.

First MB, Spitzer RL, Gibbon M, Williams JB (2001) Structured clinical interview for DSM-IV-TR Axis I disorders, Patient Edition (SCID-I/P). New York: New York State Psychiatric Institute.

Fusar-Poli P, Perez J, Broome M, Borgwardt S, Placentino A, Caverzasi E, Cortesi M, Veggiotti P, Politi P, Barale F, McGuire P (2007) Neurofunctional correlates of vulnerability to psychosis: A systematic review and meta-analysis. Neurosci Biobehav Rev 31:465-484.

Gilbert AR, Rosenberg DR, Harenski K, Spencer S, Sweeney JA, Keshavan MS (2001) Thalamic volumes in patients with first-episode schizophrenia. Am J Psychiatry 158:618-624.

Gottesman I (1991) Schizophrenia genesis: the origins of madness. New York: W. H. Freeman.

Gottesman II, Gould TD (2003) The endophenotype concept in psychiatry: etymology and strategic intentions. Am J Psychiatry 160:636-645.

Gur RE, Maany V, Mozley PD, Swanson C, Bilker W, Gur RC (1998) Subcortical MRI volumes in neuroleptic-naive and treated patients with schizophrenia. Am J Psychiatry 155:1711-1717.

Haller JW, Banerjee A, Christensen GE, Gado M, Joshi S, Miller MI, Sheline Y, Vannier MW, Csernansky JG (1997) Three-dimensional hippocampal MR morphometry with high-dimensional transformation of a neuroanatomic atlas. Radiology 202:504-510.

Hazlett EA, Buchsbaum MS, Byne W, Wei TC, Spiegel-Cohen J, Geneve C, Kinderlehrer R, Haznedar MM, Shihabuddin L, Siever LJ (1999) Threedimensional analysis with MRI and PET of the size, shape, and function of the thalamus in the schizophrenia spectrum. Am J Psychiatry 156:1190-1199.

Hollingshead AB (1975) Four factor index of social status. New Haven, CT: Yale University.

Kemether EM, Buchsbaum MS, Byne W, Hazlett EA, Haznedar M, Brickman AM, Platholi J, Bloom R (2003) Magnetic resonance imaging of mediodorsal, pulvinar, and centromedian nuclei of the thalamus in patients with schizophrenia. Arch Gen Psychiatry 60:983-991.

Kendler KS, Gruenberg AM, Kinney DK (1994) Independent diagnoses of adoptees and relatives as defined by DSM-III in the provincial and national samples of the Danish Adoption Study of Schizophrenia. Arch Gen Psychiatry 51:456-468.

Kenward MG, Roger JH (1997) Small sample inference for fixed effects from restricted maximum likelihood. Biometrics 53:983-997.

Khorram B, Lang DJ, Kopala LC, Vandorpe RA, Rui Q, Goghari VM, Smith GN, Honer WG (2006) Reduced thalamic volume in patients with chronic schizophrenia after switching from typical antipsychotic medications to olanzapine. Am J Psychiatry 163:2005-2007.

Konick LC, Friedman L (2001) Meta-analysis of thalamic size in schizophrenia. Biol Psychiatry 49:28-38.

Lawrie SM, Whalley HC, Abukmeil SS, Kestelman JN, Donnelly L, Miller P, Best JJ, Owens DG, Johnstone EC (2001) Brain structure, genetic liability, and psychotic symptoms in subjects at high risk of developing schizophrenia. Biol Psychiatry 49:811-823.

Lopez-Bendito G, Molnar Z (2003) Thalamocortical development: how are we going to get there? Nat Rev Neurosci 4:276-289.

Mamah D, Wang L, Barch D, de Erausquin GA, Gado M, Csernansky JG (2007) Structural analysis of the basal ganglia in schizophrenia. Schizophr Res 89:59-71.

McDonald C, Bullmore ET, Sham PC, Chitnis X, Wickham H, Bramon E, Murray RM (2004) Association of genetic risks for schizophrenia and bipolar disorder with specific and generic brain structural endophenotypes. Arch Gen Psychiatry 61:974-984.

Miller M, Banerjee A, Christensen G, Joshi S, Khaneja N, Grenander U, Matejic L (1997) Statistical methods in computational anatomy. Stat Methods Med Res 6:267-299.

Miller TJ, McGlashan TH, Woods SW, Stein K, Driesen N, Corcoran CM, Hoffman R, Davidson L (1999) Symptom assessment in schizophrenic prodromal states. Psychiatr Q 70:273-287.

Pakkenberg B (1992) The volume of the mediodorsal thalamic nucleus in treated and untreated schizophrenics. Schizophr Res 7:95-100.

Schulze K, McDonald C, Frangou S, Sham P, Grech A, Toulopoulou T, Walshe M, Sharma T, Sigmundsson T, Taylor M, Murray RM (2003) Hippocampal volume in familial and nonfamilial schizophrenic probands and their unaffected relatives. Biol Psychiatry 53:562-570.

Seidman LJ, Faraone SV, Goldstein JM, Goodman JM, Kremen WS, Toomey R, Tourville J, Kennedy D, Makris N, Caviness VS, Tsuang MT (1999) Thalamic and amygdala-hippocampal volume reductions in first-degree relatives of patients with schizophrenia: an MRI-based morphometric analysis. Biol Psychiatry 46:941-954.

Selemon LD, Wang L, Nebel MB, Csernansky JG, Goldman-Rakic PS, Rakic P (2005) Direct and indirect effects of fetal irradiation on cortical gray and white matter volume in the macaque. Biol Psychiatry 57:83-90.

Sim K, Cullen T, Ongur D, Heckers S (2006) Testing models of thalamic dysfunction in schizophrenia using neuroimaging. J Neural Transm 113:907-928.

Staal WG, Hulshoff Pol HE, Schnack H, van der Schot AC, Kahn RS (1998) Partial volume decrease of the thalamus in relatives of patients with schizophrenia. Am J Psychiatry 155:1784-1786.

Sullivan EV, Rosenbloom MJ, Serventi KL, Deshmukh A, Pfefferbaum A (2003) Effects of alcohol dependence comorbidity and antipsychotic medication on volumes of the thalamus and pons in schizophrenia. Am J Psychiatry 160:1110-1116.

van Erp TG, Saleh PA, Rosso IM, Huttunen M, Lonnqvist J, Pirkola T, Salonen O, Valanne L, Poutanen VP, Standertskjold-Nordenstam CG, Cannon TD (2002) Contributions of genetic risk and fetal hypoxia to hippocampal volume in patients with schizophrenia or schizoaffective disorder, their unaffected siblings, and healthy unrelated volunteers. Am J Psychiatry 159:1514-1520.

van Erp TG, Saleh PA, Huttunen M, Lonnqvist J, Kaprio J, Salonen O, Valanne L, Poutanen VP, Standertskjold-Nordenstam CG, Cannon TD (2004) Hippocampal volumes in schizophrenic twins. Arch Gen Psychiatry 61:346-353.

Wang L, Lee DY, Bailey E, Hartlein JM, Gado MH, Miller MI, Black KJ (2007) Validity of large-deformation high dimensional brain mapping of the basal ganglia in adults with Tourette syndrome. Psychiatry Res 154:181-190.

Wiegell MR, Tuch DS, Larsson HB, Wedeen VJ (2003) Automatic segmentation of thalamic nuclei from diffusion tensor magnetic resonance imaging. Neuroimage 19:391-401.

Young KA, Manaye KF, Liang C, Hicks PB, German DC (2000) Reduced number of mediodorsal and anterior thalamic neurons in schizophrenia. Biol Psychiatry 47:944-953. 